Estimating time-varying treatment effects in longitudinal studies

\author{
Wen Wei Loh ${ }^{1}$ and and Dongning Ren ${ }^{2}$ \\ ${ }^{1}$ Department of Quantitative Theory and Methods \\ Emory University \\ Atlanta \\ USA \\ ${ }^{2}$ Department of Social Psychology \\ Tilburg University \\ Tilburg \\ The Netherlands
}

This manuscript has been accepted for publication in Psychological Methods. It is posted online ahead of print. 


\begin{abstract}
Longitudinal study designs are frequently used to investigate the effects of a naturally observed predictor (treatment) on an outcome over time. Because the treatment at each time point or wave is not randomly assigned, valid inferences of its causal effects require adjusting for covariates that confound each treatment-outcome association. But adjusting for covariates which are inevitably time-varying is fraught with difficulties. On the one hand, standard regression adjustment for variables affected by treatment can lead to severe bias. On the other hand, omitting time-varying covariates from confounding adjustment precipitates spurious associations that can lead to severe bias. Thus, either including or omitting time-varying covariates for confounding adjustment can lead to incorrect inferences. In this paper, we introduce an estimation strategy from the causal inference literature for evaluating the causal effects of time-varying treatments in the presence of time-varying confounding. G-estimation of the treatment effect at a particular wave proceeds by carefully adjusting for only pre-treatment instances of all variables while dispensing with any post-treatment instances. The introduced approach has various appealing features. Effect modification by time-varying covariates can be investigated using covariate-treatment interactions. Treatment may be either continuous or noncontinuous with any mean model permitted. Unbiased estimation requires correctly specifying a mean model for either the treatment or the outcome, but not necessarily both. The treatment and outcome models can be fitted with standard regression functions. In summary, g-estimation is effective, flexible, robust, and relatively straightforward to implement.

Keywords: Causal inference; Double robust estimation; G-estimation; Post-treatment confounding; Propensity scores; Time-varying confounding
\end{abstract}




\section{Estimating time-varying treatment effects in longitudinal studies}

Longitudinal study designs are frequently employed in psychological sciences. Longitudinal data is beneficial for gaining empirical insights into the causal effects of a naturally observed predictor (henceforth termed treatment) on an outcome over time. For example, researchers may be interested in whether positive affect promotes healthy aging (Hittner et al., 2020); or whether work activities predict vocational interests (Nye et al., 2021); or whether social contact frequency enhances happiness (Ren et al., 2022). Despite the prevalence of longitudinal studies in diverse research topics, causal inferences from longitudinal data are notoriously challenging. In observational studies where treatment measured at a single time point - is not randomly assigned, valid inference of the causal effect requires adjusting for baseline confounders (herein defined as common causes) of the treatment-outcome relation. Therefore, the unconfoundedness assumption states that sufficient baseline or pre-treatment covariate information is available so that the association between treatment and outcome is unconfounded within levels of the covariates (Hernán \& Robins, 2020; Imbens \& Rubin, 2015; Steiner et al., 2010) 1

While the unconfoundedness assumption can reasonably be upheld under specific settings with a single treatment time point, the assumption is challenging to meet in a longitudinal study. In particular, the intricacies of time-varying confounding are routinely overlooked and largely unaddressed in psychology research. In a longitudinal design, many - if not all - variables inevitably change over time. These variables may include confounders of the treatment-outcome association and, therefore, need to be adjusted for. Yet adjusting for these measured variables introduces another form of bias, because these variables are simultaneously affected by an earlier treatment (variously termed

\footnotetext{
${ }^{1}$ Researchers are encouraged to carefully and thoughtfully conceive of relevant baseline covariates toward improving the plausibility and defensibility of the unconfoundedness assumption. Please see the "Practical considerations for implementing g-estimation" subsection in the Discussion section for further recommendations.
} 
treatment-induced, intermediate, post-treatment or time-dependent or varying confounding). Standard estimation methods, such as employing a single regression model for the outcome given all treatments and covariates, cannot avoid undue adjustment for measured post-treatment confounders, thus inducing spurious associations between treatment and outcome (Rosenbaum, 1984). Simply put, standard regression methods adjusting for such confounders are prone to possibly severe bias (Daniel et al., 2013, Keogh et al., 2017, Robins, 2000a).

To illustrate the challenges of confounding adjustment that time-varying covariates can engender, we will use the following hypothetical example. Suppose researchers are following a cohort of first-year college students to investigate whether participating in a social belonging program during their first year in college (treatment $A$ ) contributes to their academic success at the end of their first year (outcome $Y$ ). The program involves two sessions: an initial session at wave $t=0$, and a follow-up session at wave $t=1$. To index the time point or wave a variable is recorded, we will use a subscript; e.g., $A_{t}$ denotes whether a student participates in the session at wave $t$. The outcome variable, academic success (e.g., first-year grade point average), is assessed at the end of the study at wave $t=2.2$ The researchers thus seek to estimate the causal effects of the non-randomized treatment (participating in the social-belonging program) at the initial wave $\left(A_{0}\right)$ and the subsequent wave $\left(A_{1}\right)$ on the outcome (students' academic success; $Y$ ). The causal and temporal relations among the occurrences of the variables can be represented in a causal diagram (Elwert, 2013; Glymour et al., 2016; Grosz et al., 2020) such as that shown in Figure 13

\footnotetext{
${ }^{2}$ As is common in most longitudinal designs, the outcome is assumed to be observed at a time point after the (last) treatment measurement to rule out the possibility of reverse causation from considering contemporaneous treatment-outcome relations.

${ }^{3}$ In a causal diagram, vertices represent (occurrences of) variables and arrows represent causal paths. Hence, an implicit temporal ordering is assumed; e.g., $Y$ occurs after all other variables; $A_{1}$ occurs after $A_{0}$ and $L_{0}$. Further, each (occurrence of a) variable is assumed to be generated from an unknown function of
} 
The non-randomized treatment instances likely share common causes with the outcome, which we collectively denote by $L$. Continuing our example, one such covariate $L$ is a student's concern about their social relationships (termed as "belonging uncertainty") in their first year of college. Therefore, a student's belonging uncertainty at time $t=0\left(L_{0}\right)$ can be a common cause of whether a student participates in the program $\left(A_{0}\right.$ and $\left.A_{1}\right)$ and their academic success $(Y)$. Similarly, their belonging uncertainty at time $t=1\left(L_{1}\right)$ can affect their participation in the program $\left(A_{1}\right)$ and their academic success $(Y)$. Hence, when estimating the causal effects of both treatment instances on the outcome, both $L_{0}$ and $L_{1}$ would be adjusted for to eliminate confounding bias of the treatment-outcome relations; e.g., when regressing $Y$ on both $A_{0}$ and $A_{1}$, the covariates $L_{0}$ and $L_{1}$ would routinely be included (as statistical control variables) in the same regression. But a student's belonging uncertainty in college can change over time, and it is likely to be affected by their earlier participation in the program. Therefore, $L_{1}$ can be affected by $A_{0}$ while simultaneously being a common cause of $A_{1}$ and $Y$, making $L_{1}$ a time-varying confounder.

Adjusting for a time-varying confounder can precipitate other biases. To illustrate, we continue our hypothetical example. Suppose a student's belonging uncertainty $\left(L_{0}\right.$ and $\left.L_{1}\right)$ and academic success $(Y)$ in college are influenced by their parental socioeconomic status $(U)$. Furthermore, a student's participation in the program $\left(A_{0}\right.$ and $\left.A_{1}\right)$ are (auto)correlated due to, e.g., their physical proximity to the program location or scheduling conflicts with the sessions $(V)$. We will assume that both $U$ and $V$ are unmeasured (or "hidden") as depicted in Figure 1. It can be seen in Figure 1 that on the pathway $A_{0} \rightarrow L_{1} \leftarrow U \rightarrow Y, L_{1}$ is a so-called "collider." A collider on a pathway is defined as a variable on that pathway with two arrows pointing directly at it. Adjusting for a collider on a pathway, e.g., $L_{1}$, renders "collider (stratification) bias" (Elwert \& Winship, 2014; Greenland, 2003; Thoemmes, 2015) when estimating the effect between the endpoints, e.g., $A_{0}$ and $Y$, assuming all non-collider variables on that pathway are unadjusted for. The 
routine advice to avoid collider biases is to simply exclude the colliders from adjustment. But excluding $L_{1}$ from confounding adjustment renders other biases in the estimates of the effects of $A_{0}$ and $A_{1}$ on $Y$. First, as stated earlier, $L_{1}$ is a common cause of $A_{1}$ and $Y$. Omitting $L_{1}$ induces unmeasured confounding bias when estimating the effect of $A_{1}$ on $Y$. Second, note that $A_{1}$ is a collider along each of the following paths linking $A_{0}$ and $Y$ : (i) $A_{0} \rightarrow A_{1} \leftarrow L_{1} \rightarrow Y$; and (ii) $A_{0} \leftarrow V \rightarrow A_{1} \leftarrow L_{1} \rightarrow Y$. Omitting $L_{1}$ - while including $A_{1}$ as a predictor of $Y$ - induces collider bias along both these paths when estimating the effect of $A_{0}$ on $Y{ }^{4}$ Therefore, researchers are caught in a double bind where adjusting or not adjusting for $L_{1}$ when estimating the effects of $A_{0}$ and $A_{1}$ on $Y$ can, either way, lead to bias.

In this article, we introduce to the psychology literature a solution for this problem: g-estimation for time-varying treatments in the presence of time-varying confounding (Vansteelandt \& Sjölander, 2016). G-estimation is an established causal inferential-based approach in epidemiology and medical sciences for assessing time-varying treatment effects in longitudinal data (Daniel et al., 2013; Naimi et al., 2016; Newsome et al., 2018, Vansteelandt \& Joffe, 2014). G-estimation is among a broad class of so-called "g methods" (where the "g" stands for "generalized") developed by James Robins, with deep roots in causal inference research; see, e.g., Daniel et al. (2013), Hernán and Robins (2020), and Naimi et al. (2016) for an overview of g methods. We briefly describe how g-estimation for treatment at a single time point proceeds in two stages. In the first stage, a model for the treatment given covariates is fitted, and the predicted (or fitted) treatment is calculated. In the second stage, a model for the outcome given treatment, covariates, and the predicted treatment from the first stage, is fitted. The g-estimator is then the estimator of the treatment coefficient(s) in the outcome model. When the treatment and outcome are

\footnotetext{
4 Adjusting for the collider $A_{1}$ along the path $A_{0} \leftarrow V \rightarrow A_{1} \leftarrow L_{1} \rightarrow Y$, while omitting both $V$ and $L_{1}$ from adjustment, induces so-called "included variable bias," or "M-bias." (Ding \& Miratrix, 2015 Greenland, 2003 Thoemmes, 2015).
} 
measured at multiple time points, g-estimation of the causal effect of treatment at a particular wave proceeds by first carefully transforming the outcome, then appropriately adjusting for only pre-treatment instances of all variables to ensure that the estimates are not prone to bias due to post-treatment confounding. We will therefore describe the g-estimation strategy in detail in this paper. Furthermore, we illustrate it using a publicly available real-world dataset from a longitudinal study on the effects of media use on life satisfaction (Johannes et al., 2022). All R (R Core Team, 2021) scripts used in the simulation study and illustration are freely available online and as part of the Supplemental Online Materials: $:^{5}$

We recommend g-estimation for time-varying treatments for the following desirable features. First, covariates can be either continuous or noncontinuous, and be either time-invariant or time-varying (and thus possibly affected by treatment), with no models for the (distribution of the) covariates required for estimation. Second, effect modification by both time-invariant and time-varying covariates can be readily investigated by postulating covariate-treatment interactions in a model for the linear relationship between the treatment and the (potential) outcomes. Third, continuous and noncontinuous treatments can be readily accommodated using any mean model for the treatment. Fourth, assuming that the linear relationship between the treatment and the (potential) outcomes is correctly specified and unconfoundedness is met, the resulting g-estimator is consistent for the treatment effects when the treatment model or outcome model is correctly specified, but not necessarily both. Finally, the treatment and outcome models can be fitted using standard regression functions commonly available in statistical software and thus readily accessible to psychology researchers.

5 https://github.com/wwloh/time-varying-g-estimation 


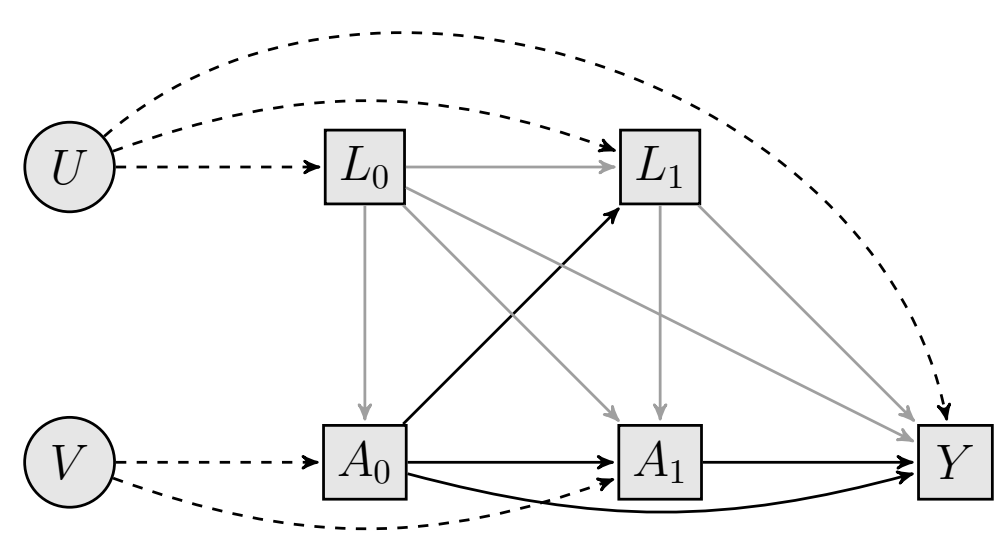

\section{Figure 1}

Causal diagram with treatment $A_{t}$ and covariate(s) $L_{t}$ observed at time points $t=0,1$, and outcome $Y$ observed at $T=2$. The subscript $t$ indexes a variable observed at time $t$.

Unmeasured baseline confounders of the covariate(s)-outcome relation(s) are represented by the common cause(s) U. Unmeasured baseline confounders of the treatment across time points are represented by the common cause(s) $V$. When unconfoundedness holds, there are no unmeasured common causes of $U$ and $V$. For visual clarity, the covariates at each time $t$ are collectively denoted by $L_{t}$ and represented by a single node. However, the effects of $L_{t}$ on each variable (drawn as gray arrows) can have different strengths. Rectangular (round) nodes denote observed (unmeasured) variables, and solid (broken) edges denote arrows emanating from observed (unmeasured) variables. 


\section{Estimating effect of treatment at a single time point}

To introduce key concepts and ideas of g-estimation for time-varying treatments, we first consider an observational study on the effect of a treatment $A$ assessed at a single time point on an end-of-study outcome $Y$. We will adopt the potential outcomes framework that facilitates definitions and interpretations of causal effects without relying on any specific statistical model. Let $Y(a)$ denote an individual's potential outcome that would have been observed under, possibly counter to actuality, treatment level $A=a .^{6}$ Continuing our hypothetical example, suppose there was only a single session of the social belonging program, where students either participated $(A=1)$ or did not participate $(A=0)$. Then $Y(1)$ (or $Y(0))$ is a student's potential academic success had they (not) participated in the program. Suppose we have sufficient baseline or pre-treatment covariate information $L$ to justify that the association between treatment and outcome is unconfounded within strata defined by $L$. Formally, we assume that $Y(a) \Perp A \mid L=l$ for all feasible treatment levels $a$ and all possible values of $L=l$, where $\Perp$ denotes (conditional) independence. Finally, we will use uppercase, e.g., $X$, to denote a random variable, and lowercase, e.g., $x$, to represent a fixed value. Let $\mathrm{E}(X \mid L=l)$ denote the conditional expectation of $X$ in the stratum with covariate values $L=l$.

\section{G-estimation in linear structural mean models}

A linear structural mean model (SMM; Robins, 1994, Vansteelandt and Joffe, 2014) is a model for the average causal effect that is conditional on the covariates and linear in an unknown (finite-dimensional) causal parameter $\psi$. It encodes the average change in potential outcomes from setting the treatment to level $a$ versus zero on the additive scale

\footnotetext{
${ }^{6}$ Throughout this paper, we maintain the stable unit treatment value assumption (SUTVA; Rubin, 1986 ) that (i) the treatment levels are well-defined with "no hidden treatment variations" among individuals experiencing the same condition, and (ii) each individual's potential outcomes are unaffected by treatments experienced by other individuals so that there is "no (treatment) interference" (Imbens \& Rubin, 2015). The causal consistency assumption (Pearl, 2010) can thus be made so that $Y=Y(A)$.
} 
amongst individuals with covariate values $L=l \cdot 7$ For example, a linear SMM which describes an average treatment effect that remains the same across all covariate levels is:

$$
\mathrm{E}\{Y(a)-Y(0) \mid L=l\}=\psi_{0} a
$$

Continuing our hypothetical example, $\psi_{0}$ encodes the average treatment effect of participating (versus not participating) in the social belonging program on academic success.

However, substantive interest is often in discerning effect heterogeneity to inform future interventions targeted at specific subgroups. Let $Z$ denote modifiers (or moderators) of the treatment effect that may be a subset of the confounders $L$ sufficient for unconfoundedness to hold. A linear SMM can include interaction terms between each modifier in $Z$ and the treatment $a$ that permits the treatment effect to differ across various levels of $Z$. For example, suppose that interest is in investigating how the treatment effect is modified by age. Such effect heterogeneity can be investigated by including an interaction term between treatment $a$ and the (grand) mean-centered variable Age:

$$
\mathrm{E}\{Y(a)-Y(0) \mid L=l\}=\left(\psi_{0}+\psi_{1} \text { Age }\right) a
$$

Here $\psi_{0}$ encodes the average treatment effect at the sample average age (when the variable Age equals zero), and $\psi_{1}$ encodes the heterogeneity due to a different value of Age.

Continuing our example, $\psi_{0}$ encodes the average treatment effect of participating (versus not participating) in the social belonging program among those at the sample average age, whereas $\psi_{1}$ encodes how the effect may differ for students with different ages (relative to the sample mean).

In general, a structural mean model describing the conditional average effect of

\footnotetext{
${ }^{7}$ We assume that a zero level for treatment encodes a meaningful pre-determined reference level such as a control condition where the treatment is absent, or the sample mean if the treatment is continuous and mean-centered.
} 
treatment on the potential outcomes given $L=l$ is:

$$
\mathrm{E}\{Y(a)-Y(0) \mid L=l\}=\psi^{\prime} z a
$$

where $\psi$ and $z$ are vectors of treatment effects and modifiers, respectively, of the same length, so that the cross-product $\psi^{\prime} z$ returns a scalar. For example, the SMM in (1) can be obtained by setting $\psi=\psi_{0}$ and $z=1$, and the SMM in (2) can be obtained by setting $\psi=\left(\psi_{0}, \psi_{1}\right)$ and $z=(1$, Age $) t^{8}$ The causal parameter $(\mathrm{s}) \psi$ in a given linear SMM (3) can be estimated using the following procedure.

1. Fit a model for the expected value of $A$, by regressing $A$ on the covariates $L$. For example, given a user-specified link function $g(\cdot)$, a model with only main effects for $L$ is:

$$
g(\mathrm{E}(A \mid L=l))=\alpha^{\prime} l
$$

Here $\alpha$ is a vector of regression coefficients for the observed values of the covariates $L=l$, and therefore of the same length as $L$. For notational simplicity, the constant intercept one is included in $L$. For continuous treatments, let $g(\mu)=\mu$ be the identity link to utilize a linear regression model. For noncontinuous treatments, one may consider other (canonical) link functions; e.g., for a binary treatment, the logit $\operatorname{link} g(\mu)=\log \{\mu /(1-\mu)\}$ leads to a logistic regression model. Here and throughout, we will refer to $\mathrm{E}(A \mid L=l)$, the conditional expectation of the treatment given the covariates $L$, as the "propensity score" (Rosenbaum \& Rubin, 1983), even though this

\footnotetext{
${ }^{8}$ We emphasize that the parameters in an SMM encode structural, i.e. causal effects of treatment defined in terms of conditional mean, i.e., average potential outcomes. Crucially, an SMM makes no parametric modeling assumptions about the functional form of the covariates (other than how they may moderate the treatment effect) or the distributions of the error terms (e.g., normality and constant variance). Under certain parametric assumptions (such as linearity), SMMs can adopt regression functional forms equivalent to standard linear regression models (Vansteelandt \& Joffe, 2014; Vansteelandt \& Sjölander, 2016). Therefore, routine statistical models familiar to most psychology researchers can be viewed as parametric representations of SMMs. We thank an anonymous reviewer for raising this point.
} 
term is commonly reserved for only binary treatments. Likewise, we refer to the regression model for the treatment in (4) as the "propensity score model." Let $\widehat{\alpha}$ denote an estimator of $\alpha$ obtained using, e.g., ordinary least squares (OLS) if a linear regression model is used or maximum likelihood estimation if a logistic regression model is used.

2. Calculate the predicted propensity score, denoted by $P=g^{-1}\left(\widehat{\alpha}^{\prime} L\right)$, for each individual by plugging in $\widehat{\alpha}$ for $\alpha$ in (4).

3. Regress the outcome on: (i) the covariates $L$; (ii) the terms $Z A$ in the SMM (3); and (iii) the corresponding terms $Z P$ obtained by substituting the treatment $A$ in the SMM by the (predicted) propensity score $P$. For example, a linear model for the outcome with only main effects for $L$ is:

$$
\mathrm{E}(Y \mid A=a, L=l)=\beta_{0}^{\prime} l+\beta_{1}^{\prime} z p+\psi^{\prime} z a .
$$

We will refer to the above model as the "outcome model." Note that the last term corresponds to the SMM (3). Calculate the OLS estimator of the causal parameter $\psi$, denoted by $\widehat{\psi}$.

Confidence intervals can be estimated using a nonparametric percentile bootstrap procedure (Efron \& Tibshirani, 1994) that randomly resamples individuals with replacement, then repeats all the above steps.

\section{Why the predicted treatment is included as a predictor of outcome}

Including the (predicted) propensity score $P$ as an additional predictor in the outcome model (5) is termed "regression adjustment for the propensity score" (D’Agostino Jr, 1998; Vansteelandt \& Daniel, 2014). When the SMM (3) is correctly specified, the resulting OLS estimator $\widehat{\psi}$ is equivalent to a g-estimator (Vansteelandt \& Joffe, 2014; Vansteelandt \& Sjölander, 2016). The central appeal of such g-estimators is its double robustness (Bang \& Robins, 2005, Kang \& Schafer, 2007; Robins, 2000b): it is 
unbiased when both the propensity score model (4) and the outcome model (5) are correctly specified, and consistent when either one is correctly specified. To see why the estimator is doubly robust, we restate the outcome model (5) as:

$$
\mathrm{E}(Y \mid A=a, L=l)=\beta_{0}^{\prime} l+\beta_{1}^{* \prime} z p+\psi^{\prime} z(a-p)
$$

where $\beta_{1}^{* \prime}=\beta_{1}+\psi$. When the SMM is correctly specified (so that the relation between the outcome $Y$ and the propensity score $P$ is correctly specified) and the propensity score model (4) is correctly specified, then $Z(A-P)$ is uncorrelated with the covariates $L$ and $Z P$. It follows from the properties of OLS estimators that $\widehat{\psi}$ is not susceptible to misspecification of the associations between outcome and covariates in the outcome model (5). In other words, a correctly-specified propensity score summarizes the covariates sufficiently to ensure unconfoundedness, thereby wholly removing any biases due to misspecifying the associations between the covariates and the outcome in (5). Even though adjustment for covariates in the outcome model is not necessary for consistent estimation, it is recommended because it may reduce residual variation and thereby increase precision (Vansteelandt \& Daniel, 2014; Vansteelandt \& Joffe, 2014).

Conversely, suppose that the outcome model (5) is correctly specified before the propensity score $P$ is added, in the sense that the associations between outcome and covariates are correctly modeled; i.e., $\mathrm{E}(Y \mid A=0, L=l)=\beta_{0}^{\prime} l$. Adding $P$ merely results in an overfitted outcome model so that the choice of a possibly misspecified propensity score model (4) does not affect the unbiasedness of the g-estimator $\widehat{\psi}$ under a correctly specified outcome model. In the next section, we will exploit such protection against model misspecification biases when either model is correctly specified to develop extensions to time-varying exposures.

\section{Continuous treatments}

The introduced g-estimation procedure permits continuous or noncontinuous treatments by accommodating any mean model for the treatment in step 1. Applied 
researchers are often interested in investigating the effects of a naturally observed focal predictor (i.e., non-randomized treatment), and this predictor of interest can be continuous or noncontinuous. For example, researchers have examined the effect of positive affect on healthy aging (Hittner et al., 2020), and the effect of social contact frequency on well-being (Ren et al., 2022). The treatments of interest (positive affect and social contact frequency, respectively) were continuous in these two settings. When the treatment is continuous, linear regression may be reasonably assumed for the propensity score model (4); see, e.g., Hirano and Imbens (2004). Further, if the predictors in (4) are also present in the outcome model (5), then the predicted propensity score $P$ is a linear combination of the predictors in the latter. In other words, the OLS estimator $\widehat{\alpha}$ results in a predicted propensity score $P$ that is perfectly collinear with the covariates in (5).9.$^{9}$ Under such a scenario, it is unnecessary to include $P$ as a predictor in the outcome model because its coefficient cannot be estimated using standard OLS. Therefore, assuming that the outcome model is correctly specified, steps 1 and 2 can be omitted from the procedure. The OLS estimator $\widehat{\psi}$ can then be calculated using just an outcome model (without $P$ ) in step 3 while preserving its double robustness (Ghosh \& Tan, 2022; Vansteelandt \& Daniel, 2014). This (under-appreciated) robustness property can make OLS estimators more attractive when the above conditions hold.

\section{Estimating the effect of a time-varying treatment}

Consider now an observational study on the effect of a time-varying treatment $A_{t}$, repeatedly assessed at time points $t=0, \ldots, T-1$, on an end-of-study outcome $Y$ assessed at time $T$. In general, for a random variable $X$, let $\bar{X}_{t}=\left(X_{0}, \ldots, X_{t}\right)$ denote the history of

\footnotetext{
${ }^{9}$ When treatment is continuous, and a linear regression model for the propensity score is assumed, then $g(\mu)=\mu$ in 4 is the identity link. It follows from an established linear algebra result for ordinary least squares estimation that the fitted (or predicted) values of a dependent variable, e.g., $P$, from a (multiple) linear regression model is an exact linear combination of, and thus perfectly collinear with, the independent variable(s), e.g., $L$.
} 
a random variable $X$ up to and including time $t$, and let its lowercase $\bar{x}_{t}=\left(x_{0}, \ldots, x_{t}\right)$ denote a fixed value. Baseline measurements of variables are recorded at the start of the study $(t=0)$, and we adopt the notational convention that any negative subscripts, e.g., $\bar{X}_{-1}=\bar{X}_{t-2}$ when $t=1$, refer to the empty or null set with no variables. Let $Y\left(\bar{a}_{t}, 0\right)$ denote the potential outcome (at time $T$ ) under a hypothetical (i.e., possibly counter to actuality) treatment program or regimen in which treatment $\bar{A}_{t}$ up to and including a given time $t<T$ is set to $\bar{a}_{t}$, and zero thereafter from time points $t+1$ to $T-1$. Let $Y(0)$ denote the potential outcome when the treatment is set to zero at all time points $t=0, \ldots, T-1$; and let $Y\left(\bar{a}_{t}\right)$ denote the potential outcome when $t=T-1$. Throughout, we continue to make the causal consistency assumption that $Y=Y\left(\bar{A}_{T-1}\right)$. Continuing our hypothetical example with $T=2, Y\left(\bar{a}_{t=0}=1,0\right)$ is a student's potential academic success had they participated in the initial session of the social belonging program at wave $t=0$ (so that $\bar{a}_{0}=a_{0}=1$ ), and not participated in the subsequent session at wave $t=1$ (so that $\left.a_{1}=0\right)$. Similarly, $Y(0)$ is a student's potential academic success had they, possibly counter to actuality, not participated in both sessions. In general, $Y\left(\bar{a}_{1}\right)$ is a student's potential academic success had their participation in both sessions, possibly counter to actuality, been at the levels $\bar{a}_{1}=\left(a_{0}, a_{1}\right)$.

Suppose that at each time $t=0, \ldots, T-1$, we have available sufficient covariate information so that the association between treatment at that time and outcome is unconfounded within levels of the covariate history up to (and including) time $t$ and of the treatment history up to (and including) time $t-1 \sqrt{10}$ Formally, we assume that $Y\left(\bar{a}_{t}, 0\right) \Perp A_{t} \mid \bar{L}_{t}=\bar{l}_{t}, \bar{A}_{t-1}=\bar{a}_{t-1}$, for all feasible treatment regimens $\bar{a}_{t}$ and all possible values of $\bar{L}_{t}=\bar{l}_{t}$ and at all times $t$. This assumption is implied in Figure 1 by the absence

\footnotetext{
${ }^{10}$ In addition to time-invariant covariates, researchers should also seek to measure all pertinent time-varying covariates that are treatment-outcome confounders - and possibly affected by treatment throughout the longitudinal study to provide a more defensible justification of the unconfoundedness assumptions. Please see the "Practical considerations for implementing g-estimation" subsection in the Discussion section for further recommendations.
} 
of any hidden common causes of each $A_{t}-Y$ relation for $t=0, \ldots, T-1$. When the repeated treatment and the repeated covariate measurements are (auto)correlated due to hidden $U$ and $V$, respectively, the unconfoundedness assumption extends to include no hidden common causes of $U$ and $V$. Finally, note that unconfoundedness holds even if $U$ includes a common cause of the covariates $L_{t}$ and the outcome $Y$ (as represented in Figure 1 by the arrow from $U$ to $Y$ ).

\section{Linear structural nested mean models}

A linear structural nested mean model (SNMM; Robins, 1994) describes, at each time $t$, the effect of setting treatment $A_{t}$ to level $a_{t}$ versus zero, with the treatment at all later times (i.e., at times $t+1$ to $T-1$ ) hypothetically set to zero, within strata defined by the covariate history up to that time and treatment history before that time. For example, an SNMM describing the conditional average effect of treatment at time $t$ on the potential outcomes (at time $T$ ) given $\bar{L}_{t}=\bar{l}_{t}$ and $\bar{A}_{t-1}=\bar{a}_{t-1}$ is:

$$
\mathrm{E}\left\{Y\left(\bar{a}_{t}, 0\right)-Y\left(\bar{a}_{t-1}, 0\right) \mid \bar{L}_{t}=\bar{l}_{t}, \bar{A}_{t-1}=\bar{a}_{t-1}\right\}=\psi_{t, 0} a_{t}
$$

The above SNMM postulates an average treatment effect $\psi_{t, 0}$ on the end-of-study outcome that is different at each time $t$ but is constant across levels of the covariate history and the

previous (hypothetical) treatment values $\bar{a}_{t-1}$. Continuing our hypothetical example, $\psi_{t=0,0}$ encodes the average treatment effect of participating in the initial session at time $t=0$ on academic success; whereas $\psi_{t=1,0}$ encodes the average treatment effect of participating in the subsequent session at time $t=1$ on academic success, conditional on the initial belonging program status $\left(A_{0}=a_{0}\right)$ at $t=0$ and prior covariate values $\left(L_{0}\right.$ and $\left.L_{1}\right)$. Alternatively, an SNMM that allows for the treatment effect at each time $t$ to be modified by a time-invariant covariate, e.g., Age, is:

$$
\mathrm{E}\left\{Y\left(\bar{a}_{t}, 0\right)-Y\left(\bar{a}_{t-1}, 0\right) \mid \bar{L}_{t}=\bar{l}_{t}, \bar{A}_{t-1}=\bar{a}_{t-1}\right\}=\left(\psi_{t, 0}+\psi_{t, 1} \text { Age }\right) a_{t} .
$$

Similar to (2), the causal parameter $\psi_{t, 1}$ encodes the average effect of treatment $A_{t}$ on outcome $Y$ (at time $T$ ) that depends on Age. Another example of an SNMM is to permit 
effect modification by time-varying covariates, e.g., belonging uncertainty ("BelUnc") at time $t$ :

$$
\mathrm{E}\left\{Y\left(\bar{a}_{t}, 0\right)-Y\left(\bar{a}_{t-1}, 0\right) \mid \bar{L}_{t}=\bar{l}_{t}, \bar{A}_{t-1}=\bar{a}_{t-1}\right\}=\left(\psi_{t, 0}+\psi_{t, 1} \text { Age }+\psi_{t, 2} \text { BelUnc }_{t}\right) a_{t} .
$$

The causal parameter $\psi_{t, 2}$ encodes the average effect of treatment $A_{t}$ on outcome $Y$ (at time $T$ ) that depends on the observed belonging uncertainty at time $t$. Continuing our example, $\psi_{t=0,2}$ encodes the heterogeneity in the average treatment effect of participating in the initial session at time $t=0$ due to different levels of baseline belonging uncertainty (at $t=0$ ), whereas $\psi_{t=1,2}$ encodes the heterogeneity in the average treatment effect of participating in the subsequent session at time $t=1$ due to different levels of the intervening belonging uncertainty (at $t=1$ ). Using the illustration in a later section, we provide an example of how to include a time-varying covariate as an effect modifier in a linear SNMM.

In general, a structural nested mean model describing the conditional average effect of treatment on the potential outcomes is:

$$
\mathrm{E}\left\{Y\left(\bar{a}_{t}, 0\right)-Y\left(\bar{a}_{t-1}, 0\right) \mid \bar{L}_{t}=\bar{l}_{t}, \bar{A}_{t-1}=\bar{a}_{t-1}\right\}=\psi_{t}^{\prime} z_{t} a_{t},
$$

where $\psi_{t}$ and $z_{t}$ are vectors of treatment effects and modifiers, respectively, of the same length so that their cross-product returns a scalar. For example, the SNMM in (6) can be obtained by setting $\psi_{t}=\psi_{t, 0}$ and $z_{t}=1$; the SNMM in (7) can be obtained by setting $\psi_{t}=\left(\psi_{t, 0}, \psi_{t, 1}\right)$ and $z_{t}=(1$, Age $)$; and the SNMM in (8) can be obtained by setting $\psi_{t}=\left(\psi_{t, 0}, \psi_{t, 1}, \psi_{t, 2}\right)$ and $z_{t}=\left(1\right.$, Age, BelUnc $\left.{ }_{t}\right)$. Therefore, researchers can use various posited linear SNMMs to meticulously investigate multiple short- and long-term causal effects - possibly modified by time-varying covariates - at different time points or waves.

Therefore, the sequence of SNMMs indexed by (treatment) times $t=0, \ldots, T-1$ describes the average effects of a time-varying treatment on the outcome under various hypothetical treatment regimens. When causal consistency holds, at each time $t, Y\left(\bar{A}_{t}, 0\right)$ is the observed outcome if the hypothetical treatment regimen agreed with the observed 
treatment history $\bar{A}_{t}$ up to time $t$, and was zero after that. The SNMM at each time $t$ thus encodes the average effect of treatment at that time on the potential outcome $Y\left(\bar{A}_{t}, 0\right)$, while considering $\bar{L}_{t}$ and $\bar{A}_{t-1}$ as pre-treatment covariates. This construction induces a sequence of SNMMs so that the cumulative average effect of all occurrences of the treatment $\bar{A}_{T-1}$ is $\sum_{t=0}^{T-1} \psi_{t}^{\prime} Z_{t} A_{t}$. In other words, to determine the potential outcome $Y\left(\bar{A}_{t}, 0\right)$ at a particular time $t$, one can iteratively subtract the treatment effects encoded by the SNMM at later times (from $t+1$ to $T-1$ ). We exploit this additive property to develop g-estimators of the causal parameters encoded in the SNMMs.

Before developing the g-estimation procedure, we offer an intuitive explanation under the most straightforward possible setting with treatment at two waves, as shown in Figure 1, for an outcome observed at time $T=2$. Under causal consistency, the observed outcome is the potential outcome under the observed treatment program; i.e., $Y=Y\left(\bar{A}_{1}\right)$. Under the SNMM $(6)$, the potential outcome $Y\left(\bar{A}_{0}, 0\right)$ can be readily determined by subtracting $\psi_{t=1,0} A_{1}$ from $Y$, i.e., $Y\left(\bar{A}_{0}, 0\right)=Y-\psi_{t=1,0} A_{1}=Y\left(\bar{A}_{1}\right)-\psi_{t=1,0} A_{1}$. This operation can be viewed as a structural transformation where the arrow from $A_{1}$ to $Y$ is removed and the outcome replaced by the potential outcome $Y\left(\bar{A}_{0}, 0\right)$. Using path-tracing rules (Wright, 1934), the (remaining) effect of $A_{0}$ on $Y$ is the combined effect along the paths $A_{0} \rightarrow Y$ and $A_{0} \rightarrow L_{1} \rightarrow Y$ which do not intersect $A_{1}$; this is concisely encoded by $\psi_{t=0,0} A_{0}$ under the SNMM (6). The potential outcome $Y(0)$ can then be determined by subtracting $\psi_{t=0,0} A_{0}$ from the potential outcome $Y\left(\bar{A}_{0}, 0\right)$, i.e., $Y(0)=Y\left(\bar{A}_{0}, 0\right)-\psi_{t=0,0} A_{0}=Y-\psi_{t=1,0} A_{1}-\psi_{t=0,0} A_{0}$. This knowledge will be crucial to the estimation procedure developed in the next section.

\section{G-estimation in linear structural nested mean models}

In this section, we describe how to obtain g-estimators of $\psi_{t}, t=0, \ldots, T-1$, under a given SNMM. Suppose we could obtain unbiased predictions of the counterfactual outcomes $Y\left(\bar{A}_{t}, 0\right)$ at each time $t=0, \ldots, T-1$, henceforth denoted by $R_{t+1}$. In that case, we could apply the estimation procedure described in the previous section (for the 
treatment at a single time point) with $R_{t+1}, A_{t}$, and $\left(\bar{L}_{t}, \bar{A}_{t-1}\right)$ in place of $Y, A$ and $L$, respectively. The estimation procedure, therefore, involves repeated application of the procedure to the residualized outcomes $R_{t+1}$ for the counterfactuals $Y\left(\bar{A}_{t}, 0\right)$. Because each application of the procedure results in a doubly robust estimator of $\psi_{t}$, the resulting g-estimator preserves the double robustness property: it is consistent when either the propensity score model is correctly specified or the association between $R_{t+1}$ and the history $\left(\bar{L}_{t}, \bar{A}_{t-1}\right)$ is correctly modeled, and unbiased when both are correct.

We describe a g-estimator of the time-varying treatments $\bar{A}_{t}$ on outcome $Y$ (at time T) as follows. For $t=T-1, \ldots, 0$ in turn:

1. Fit a propensity score model for the expected value of $A_{t}$, by regressing $A_{t}$ on the covariate history $\bar{L}_{t}$, and treatment history $\bar{A}_{t-1}$. For example, a model with main effects only is:

$$
g\left(\mathrm{E}\left(A_{t} \mid \bar{L}_{t}=\bar{l}_{t}, \bar{A}_{t-1}=\bar{a}_{t-1}\right)\right)=\alpha_{t, 0}^{\prime} \bar{l}_{t}+\alpha_{t, 1}^{\prime} \bar{a}_{t-1} .
$$

Here $\alpha_{t, 0}$ is a vector of regression coefficients for the observed values of the covariates $\bar{L}_{t}=\bar{l}_{t}$, and therefore of the same length as $\bar{L}_{t}$. Similarly, $\alpha_{t, 1}$ is a vector of regression coefficients (of length $t$ ) for the observed values of the previous treatments $\bar{A}_{t-1}=\bar{a}_{t-1}$. Let $\alpha_{t}=\left(\alpha_{t, 0}, \alpha_{t, 1}\right)$ denote the regression coefficients collectively, and let $\widehat{\alpha}_{t}$ denote an estimator of $\alpha_{t}$.

2. Calculate the predicted propensity score, denoted by $P_{t}=g^{-1}\left(\widehat{\alpha}_{t, 0}^{\prime} \bar{L}_{t}+\widehat{\alpha}_{t, 1}^{\prime} \bar{A}_{t-1}\right)$, for each individual by plugging in $\widehat{\alpha}_{t}$ for $\alpha_{t}$ in $(10)$.

3. If $t=T-1$, set the counterfactual outcome, henceforth denoted by $R_{t+1}$, to its observed value $R_{t+1}=Y$.

4. Regress the outcome $R_{t+1}$ on: (i) the covariate history $\bar{L}_{t}$; (ii) the treatment history before that time $\bar{A}_{t-1}$; (iii) the terms $Z_{t} A_{t}$ in the SNMM (9); and (iv) the corresponding terms $Z_{t} P_{t}$ obtained by substituting the treatment $A_{t}$ in the SNMM by 
the (predicted) propensity score $P_{t}$. For example, an outcome model with only main effects for $\bar{L}_{t}$ and $\bar{A}_{t-1}$ is:

$$
\mathrm{E}\left(R_{t+1} \mid \bar{L}_{t}=\bar{l}_{t}, \bar{A}_{t}=\bar{a}_{t}\right)=\beta_{t, 0}^{\prime} \bar{l}_{t}+\beta_{t, 1}^{\prime} \bar{a}_{t-1}+\beta_{t, 2}^{\prime} z_{t} p_{t}+\psi_{t}^{\prime} z_{t} a_{t}
$$

Calculate the OLS estimator of $\psi_{t}$, denoted by $\widehat{\psi}_{t}$.

It follows from the previous section (for a single time point) that if the outcome model (11) is correctly specified, then the estimator $\widehat{\psi}_{t}$ is consistent for $\psi_{t}$. But if the outcome model (11) is misspecified, then adjusting for the predicted propensity score $P_{t}$ - when the propensity score model 10 is correctly specified - continues to yield an estimator $\widehat{\psi}_{t}$ that is consistent for $\psi_{t}$.

5. If $t>0$, determine the residualized outcome as $R_{t}=R_{t+1}-\widehat{\psi}_{t}^{\prime} Z_{t} A_{t}$.

When $t=T-1$, this step removes the effect of the treatment $A_{T-1}$ on the outcome $Y$ encoded by $\psi_{T-1}^{\prime} Z_{T-1}$ in the SNMM (9); otherwise, it removes the effect of the treatment $A_{t}$ on the residualized outcome $R_{t+1}=Y-\sum_{s=t+1}^{T-1} \psi_{s}^{\prime} Z_{s} A_{s}$.

Repeating all the above steps for $t=T-1, \ldots, 0$, in turn, yields g-estimators of the

treatment effects $\widehat{\psi}_{t}, t=T-1, \ldots, 0$. Confidence intervals can be estimated using a nonparametric percentile bootstrap procedure that randomly resamples individuals with replacement and then repeats all the above steps.

\section{Time-varying outcomes}

Now suppose that the outcome $Y_{s}$ is observed not only at the final time point $s=T$ but at intervening time points $s=1, \ldots, T-1$, and at baseline $s=0$. In the following, consider the outcome $Y_{s}$ at each time $s=1, \ldots, T$, in turn as the end-of-study outcome. The previous results extend immediately to such time-varying outcomes by considering the outcome $Y_{s}$ at each time point $s$ as the end-of-study outcome (previously $\left.Y\right)$. Let $Y_{s}\left(\bar{a}_{t}, 0\right)$ denote the potential outcome (at time $s$ ) where $\bar{A}_{t}$ up to (and including) a given time $t<s$ is set to $\bar{a}_{t}$, and zero thereafter from time points $t+1$ to $s-1$. Suppose that at each time 
$t=0, \ldots, T-1$, we have available sufficient covariate information so that the association between treatment at time $t$ and the outcome at time $s$ is unconfounded within levels of the covariate history up to (and including) time $t$ and of the treatment history up to (and including) time $t-1$. For notational convenience, we will include the outcome history $\bar{Y}_{t}$ in the covariate history $\bar{L}_{t}$ up to time $t$ to control for confounding due to lagged effects by all previous outcomes. Formally, we assume that $Y_{s}\left(\bar{a}_{t}, 0\right) \Perp A_{t} \mid \bar{L}_{t}=\bar{l}_{t}, \bar{A}_{t-1}=\bar{a}_{t}$ for all feasible treatment regimes $\bar{a}_{t}$ and all possible values of $\bar{L}_{t}=\bar{l}_{t}$ and at all times $t$. We continue to make the causal consistency assumption that $Y_{s}=Y_{s}\left(\bar{A}_{s-1}\right)$.

An SNMM must then be postulated for the outcome at each time point $s$. For example, the conditional average effect of treatment at time $t$ on the potential outcomes (at time $s$ ) given $\bar{L}_{t}=\bar{l}_{t}$ and $\bar{A}_{t-1}=\bar{a}_{t-1}$ can be described using the SNMM analogous to (60):

$$
\mathrm{E}\left\{Y_{s}\left(\bar{a}_{t}, 0\right)-Y_{s}\left(\bar{a}_{t-1}, 0\right) \mid \bar{L}_{t}=\bar{l}_{t}, \bar{A}_{t-1}=\bar{a}_{t-1}\right\}=\psi_{s, t, 0} a_{t}
$$

Continuing our hypothetical example, suppose that academic success was measured not only at the end of the first year (now denoted by $Y_{2}$ ) but before the session at time $t=1$ (thus denoted by $Y_{1}$ ). Then $\psi_{s=1, t=0,0}$ encodes the average treatment effect of participating in the initial session at time $t=0$ on academic success at time $s=1$. Similarly, the SNMM analogous to $(8)$ is:

$$
\mathrm{E}\left\{Y_{s}\left(\bar{a}_{t}, 0\right)-Y_{s}\left(\bar{a}_{t-1}, 0\right) \mid \bar{L}_{t}=\bar{l}_{t}, \bar{A}_{t-1}=\bar{a}_{t-1}\right\}=\left\{\psi_{s, t, 0}+\psi_{s, t, 1} \text { Age }+\psi_{s, t, 2} \text { BelUnc }_{t}\right\} a_{t} .
$$

Continuing our example, $\psi_{s=1, t=0,1}$ and $\psi_{s=1, t=0,2}$ encode the heterogeneity in the average treatment effect of participating in the initial session at time $t=0$ on academic success at time $s=1$ due to different ages and levels of baseline belonging uncertainty (at $t=0)$, respectively.

In general, a structural nested mean model describing the conditional average effect of treatment on the potential outcomes is:

$$
\mathrm{E}\left\{Y_{s}\left(\bar{a}_{t}, 0\right)-Y_{s}\left(\bar{a}_{t-1}, 0\right) \mid \bar{L}_{t}=\bar{l}_{t}, \bar{A}_{t-1}=\bar{a}_{t-1}\right\}=\psi_{s, t}^{\prime} z_{s, t} a_{t}
$$


where $\psi_{s, t}$ and $Z_{s, t}$ are vectors of treatment effects and modifiers, respectively, of the same length so that their cross-product returns a scalar. For example, the SNMM in 12 can be obtained by setting $\psi_{s, t}=\psi_{s, t, 0}$ and $Z_{s, t}=1$; and the SNMM in 13 can be obtained by setting $\psi_{s, t}=\left(\psi_{s, t, 0}, \psi_{s, t, 1}, \psi_{s, t, 2}\right)$ and $Z_{t}=(1$, Age, BelUnc $t)$.

The g-estimator of the time-varying treatments $\bar{A}_{t}$ on outcome $Y_{s}$ (at a given time $s$ ) can be obtained by carrying out the following steps for each of $t=s-1, \ldots, 0$ in turn:

1. Fit a propensity score model for treatment at time $t$ by regressing the treatment on all causally and temporally preceding variables, such as 10 following step 1 in the preceding section.

2. Calculate the predicted propensity score $P_{t}$ by plugging in the propensity score model coefficient estimates for their unknown values following step 2 in the preceding section.

3. If $t=s-1$, set the outcome, henceforth denoted by $R_{s, t+1}$, to its observed value $R_{s, t+1}=Y_{s}$.

4. Regress $R_{s, t+1}$ on: (i) all variables causally and temporally preceding the treatment $A_{t}$ (i.e., the explanatory variables in the propensity score model in step 1 above); (ii) the terms $Z_{s, t} A_{t}$ in the SNMM (14), where $Z_{s, t}$ are the treatment effect modifiers (such as Age in the hypothetical example); and (iii) the corresponding terms $Z_{s, t} P_{t}$ obtained by substituting the treatment $A_{t}$ in the SNMM by the predicted propensity score $P_{t}$. For example, an outcome model analogous to (11), with only main effects for the covariate history $\left(\bar{L}_{t}\right)$, outcome history $\left(\bar{Y}_{t}\right)$, and treatment history $\left(\bar{A}_{t-1}\right)$, is: $\mathrm{E}\left(R_{s, t+1} \mid \bar{L}_{t}=\bar{l}_{t}, \bar{Y}_{t}=\bar{y}_{t}, \bar{A}_{t}=\bar{a}_{t}\right)=\beta_{s, t, 0}^{\prime} \bar{l}_{t}+\beta_{s, t, 1}^{\prime} \bar{y}_{t}+\beta_{s, t, 2}^{\prime} \bar{a}_{t-1}+\beta_{s, t, 3}^{\prime} z_{s, t} p_{t}+\psi_{s, t}^{\prime} z_{s, t} a_{t}$.

Calculate the OLS estimator of $\psi_{s, t}$, denoted by $\widehat{\psi}_{s, t}$.

5. If $t>0$, determine the residualized outcome as $R_{s, t}=R_{s, t+1}-\widehat{\psi}_{s, t}^{\prime} Z_{s, t} A_{t}$. 
When $t=s-1$, this step removes the effect of the treatment $A_{s-1}$ on the outcome $Y_{s}$ encoded by $\psi_{s, s-1}^{\prime} Z_{s, s-1}$ in the SNMM (14); otherwise, it removes the effect of the treatment $A_{t}$ on the residualized outcome $R_{s, t+1}=Y_{s}-\sum_{u=t+1}^{s-1} \widehat{\psi}_{s, u}^{\prime} Z_{s, u} A_{u}$.

Repeating all the above steps for each of $t=s-1, \ldots, 0$, in turn, yields g-estimators of the treatment effects $\widehat{\psi}_{s, t}, t=s-1, \ldots, 0$. Confidence intervals can be estimated using a nonparametric percentile bootstrap procedure that randomly resamples individuals with replacement and then repeats all the steps.

\section{Simulation study}

We conducted a Monte Carlo simulation study to empirically demonstrate the unbiasedness of the introduced g-estimator of time-varying treatment effects. The study was carried out under the setting shown in Figure 1, with a non-randomized binary treatment measured at the first two time points, the outcome measured at the last time point, and different time-invariant and time-varying treatment-outcome confounders. To emphasize the g-estimator's double robustness property, we generated each simulated dataset wherein the treatments were based on linear and additive propensity score models, whereas the (post-treatment) outcome was based on a model with complicated non-linear functions of the predictors. We then fitted to each simulated dataset linear and additive propensity score and outcome models so that the propensity score model was correctly specified, but the outcome model was incorrectly specified. For comparison, we considered

two other estimators that relied only on linear and additive outcome regression models and were prone to model misspecification biases. We used 1000 simulated datasets with sample sizes of 100, 400, and 1600 to investigate the (lack of) biases under various scenarios.

\section{Data-generating process}

Each simulated dataset was generated following Figure 1 by carrying out the steps below. 
1. Generate the following variables at time $t=0$ :

$$
\begin{aligned}
U, V & \sim \mathcal{N}(0,1) \\
L_{j, 0} & =U+\epsilon^{L_{j, 0}} ; \epsilon^{L_{j, 0}} \sim \mathcal{N}(0,1) ; j=1, \ldots, 6 \\
A_{0}^{*} & =\nu_{0}^{A_{0}} V+\sum_{j=1}^{6} \nu_{j}^{A_{0}} L_{j, 0} \\
A_{0} & \sim \operatorname{Bernoulli}\left\{\operatorname{expit}\left(A_{0}^{*}\right)\right\} \\
Y_{0}^{*} & =\nu_{0}^{Y_{0}} U+\sum_{j=1}^{6} \nu_{j}^{Y_{0}} L_{j, 0}+\epsilon^{Y_{0}} ; \epsilon^{Y_{0}} \sim \mathcal{N}(0,1) \\
Y_{0} & =\frac{Y_{0}^{*}-\widehat{\mathrm{E}}\left(Y_{0}^{*}\right)}{\widehat{\sigma}\left(Y_{0}^{*}\right)} .
\end{aligned}
$$

The variable $U$ was a common cause of the covariates and outcome over time, while the variable $V$ was a common cause of the treatment over time; both were hidden from the observed data to induce unmeasured confounding. The baseline outcome was a linear and additive function of the covariates and their common cause $U$. We standardized the baseline outcomes using their sample mean $\widehat{\mathrm{E}}\left(Y_{0}^{*}\right)$ and sample standard deviation $\widehat{\sigma}\left(Y_{0}^{*}\right)$. We set the values of the other coefficients to $\nu_{0}^{A_{0}}=c_{1} ; \nu_{j}^{A_{0}}=j / 8, j=1, \ldots, 6 ; \nu_{0}^{Y_{0}}=c_{2} ; \nu_{j}^{Y_{0}}=\exp (j / 8), j=1, \ldots, 6$.

2. Generate the following variables at time $t=1$ :

$$
\begin{aligned}
L_{j, 1} & =L_{j, 0} ; j=1, \ldots, 4 \\
L_{j, 1}^{*} & =\nu_{0}^{L_{j, 1}} U+\sum_{k=1}^{6} \nu_{k}^{L_{j, 1}} L_{k, 0}+\nu_{0}^{L_{j, 7}} U Y_{0}+\epsilon^{L_{j, 1}} ; \epsilon^{L_{j, 1}} \sim \mathcal{N}(0,1) ; j=5,6 \\
L_{j, 1} & =\frac{L_{j, 1}^{*}-\widehat{\mathrm{E}}\left(L_{j, 1}^{*}\right)}{\widehat{\sigma}\left(L_{j, 1}^{*}\right)}+\gamma_{j} A_{0} ; j=5,6 \\
A_{1}^{*} & =\nu_{0}^{A_{1}} V+\sum_{j=1}^{6} \nu_{j}^{A_{1}} L_{j, 1}+\nu_{7}^{A_{1}} Y_{0} \\
A_{1} & \sim \operatorname{Bernoulli}\left\{\operatorname{expit}\left(A_{1}^{*}\right)\right\} .
\end{aligned}
$$

The first four covariates (indexed by $j=1, \ldots, 4$ ) were time-invariant covariates, whereas the remaining two covariates were time-varying and affected by the earlier 
outcome $Y_{0}$ and treatment $A_{0}$. Each time-varying covariate was first generated as a linear function of the time-invariant covariates, the earlier outcome $Y_{0}$, their common cause $U$, and an interaction term $U Y_{0}$. It was then standardized using its sample mean and variance, and an additive treatment effect was applied so that the covariate was a non-linear function of its predictors. The treatment $A_{1}$ depended directly on the unmeasured confounder $V$ (and was thus correlated with the earlier treatment $\left.A_{0}\right)$, the baseline outcome $Y_{0}$, and both time-invariant and time-varying covariates. The treatment $A_{1}$ was a linear and additive function of its predictors. We set the values of the coefficients to $\nu_{0}^{L_{j, 1}}=\nu_{0}^{L_{j, 7}}=c_{2} ; \nu_{k}^{L_{j, 1}}=\exp (k / 8), k=1, \ldots, 6 ; \gamma_{5}=$ $0.35 ; \gamma_{6}=0.7 ; \nu_{0}^{A_{1}}=c_{1} ; \nu_{j}^{A_{1}}=j / 8, j=1, \ldots, 6 ; \nu_{7}^{A_{1}}=0.7$.

3. Generate the outcome at time $t=2$ :

$Y=\nu_{0}^{Y} U+\sum_{j=1}^{6} \nu_{j}^{Y} L_{j, 1}+\nu_{7}^{Y} Y_{0} \exp (U / 2)+\nu_{8}^{Y} \sqrt{\left|\prod_{j=1}^{4} L_{j, 1}\right|}+\nu_{9}^{Y} \min \left\{\left(L_{4,1} Y_{0}\right)^{2}, 3.84\right\}+\gamma_{1} A_{1}+\epsilon^{Y} ;$ where $\epsilon^{Y} \sim \mathcal{N}(0,1)$. The outcome was a non-linear function of the time-invariant covariates, the earlier outcome $Y_{0}$, and their common cause $U$. In particular, the outcome was based on complicated, arbitrary functions of its predictors intentionally designed to illustrate the g-estimator's double robustness property. The outcome was affected directly by the treatment $A_{1}$ and the time-varying covariates $L_{j, 1}, j=5,6$ at the previous time point $(t=1)$. Moreover, the outcome was indirectly affected by the earlier treatment $A_{0}$ via both intervening time-varying covariates. Hence, using the product-of-coefficients method (Baron \& Kenny, 1986, Duncan, 1966), the effect of $A_{1}$ on $Y$ was $\gamma_{1}$, whereas the effect of $A_{0}$ on $Y$ was $\sum_{j=5}^{6} \gamma_{j} \nu_{j}^{Y}$. We set the values of the coefficients to $\nu_{0}^{Y}=\nu_{7}^{Y}=c_{2} ; \nu_{j}^{Y}=\exp (j / 8), j=1, \ldots, 4 ; \nu_{5}^{Y}=1 ; \nu_{6}^{Y}=0.5 ; \nu_{8}^{Y}=\nu_{9}^{Y}=1 ; \gamma_{1}=0.7$.

We considered settings where: (i) either $\left(c_{1}, c_{2}\right)=(0,0)$, or $\left(c_{1}, c_{2}\right)=(0.7, \exp (0.7))$, with the former corresponding to no unmeasured confounding by $U$ and $V$; and (ii) the 
sample size was either 100,400 , or 1600.11

\section{Estimators under comparison}

We assumed a correctly specified SNMM corresponding to (6), so that the time-varying treatment effects of $A_{0}$ and $A_{1}$ on $Y$ were encoded by the causal parameters $\psi_{0,0}$ and $\psi_{1,0}$, respectively. We considered three different estimators of $\psi_{0,0}$ and $\psi_{1,0}$ for each simulated dataset.

1. The g-estimation procedure as described, assuming propensity score and outcome models with only main effects for the covariate (and treatment) histories. The assumed outcome model was thus misspecified due to the omitted (functions of the) interactions between the baseline covariates and outcome at time $t=0$. We utilized a logistic regression model for the correctly-specified propensity score model. We will refer to this estimator simply as "DR g-estimator," where the "DR" stands for doubly robust.

2. A simplified g-estimator using only outcome models (with only main effects for the covariates and treatments) without adjusting for propensity scores. This estimator was used to demonstrate how an incorrectly specified outcome model - which did not include the (predicted) propensity scores as a form of protection against model misspecification biases - can lead to biased estimators. We will refer to this estimator as "Outcome only."

3. A single regression model for the outcome given only main effects for all causally and temporally preceding (occurrences of the) variables. We used the coefficients of $A_{0}$ and $A_{1}$ as estimators of $\psi_{0,0}$ and $\psi_{1,0}$, respectively. Such an approach is routinely

\footnotetext{
${ }^{11}$ We considered three sample sizes for illustrative purposes. We chose $n=1600$ following the sample sizes in the applied example. We also chose a smaller sample size $(n=400)$ similar to many longitudinal studies in practice. The smallest sample size $(n=100)$ represented the lower range of sample sizes encountered in longitudinal studies in psychological research.
} 
adopted for simultaneously estimating multiple treatment effects on the same outcome. We will refer to this estimator as "Single regression." Because this regression model corresponded to the fitted outcome model for $t=T-1$ in the previous "Outcome only" estimator, the estimators of $\psi_{1,0}$ using both these methods would thus be identical. However, the estimator of $\psi_{0,0}$ would differ due to time-varying confounding by $L_{5,1}$ and $L_{6,1}$.

\section{Results}

The results for 1000 simulated datasets under each setting are shown in Table 1 . When there was no unmeasured confounding, the single regression model and the g-estimator using an outcome model only, i.e., without a propensity score model, yielded biased estimates as expected due to the misspecified associations between the covariates and the outcome. When there was unmeasured confounding, the magnitude of the biases was exacerbated by the collider biases (along paths described in the introduction). For the single regression model, one could argue that the coefficient of $A_{0}$ in such a model is not a consistent estimator of $\psi_{0,0}$, because the regression coefficient encodes only the "direct" effect of $A_{0}$ on $Y$ that is not transmitted via the intervening time-varying confounders $L_{5,1}$ and $L_{6,1}$ (both at time $t=1$ ). But even if one considered the regression coefficient of $A_{0}$ as an estimator of such a direct effect, whose true value in this study was zero, the estimator would nonetheless be biased. The biases resulted from conditioning on the colliders $L_{5,1}$ and $L_{6,1}$ along the paths linking $A_{0}$ and $Y: A_{0} \rightarrow L_{5,1} \leftarrow U \rightarrow Y$ and $A_{0} \rightarrow L_{6,1} \leftarrow U \rightarrow Y$, respectively. Finally, the introduced DR g-estimator was unbiased for the causal parameters $\psi_{0,0}$ and $\psi_{1,0}$ notwithstanding the misspecified outcome model and presence of hidden confounding by $U$ and $V$. 


\section{Table 1}

Empirical biases of estimates of the treatment effects $\psi_{0,0}$ and $\psi_{1,0}$. In addition, the empirical standard errors (ESE) and root mean squared error (RMSE) are also shown.

\begin{tabular}{|c|c|c|c|c|c|c|c|c|}
\hline \multirow[b]{2}{*}{ Sample size } & \multirow[b]{2}{*}{ Unmeasured confounding } & \multirow[b]{2}{*}{ Estimation Method } & \multicolumn{2}{|c|}{ Bias } & \multicolumn{2}{|c|}{ ESE } & \multicolumn{2}{|c|}{ RMSE } \\
\hline & & & $\psi_{0,0}$ & $\psi_{1,0}$ & $\psi_{0,0}$ & $\psi_{1,0}$ & $\psi_{0,0}$ & $\psi_{1,0}$ \\
\hline 100 & FALSE & DR g-estimator & -0.01 & 0.02 & 0.52 & 0.54 & 0.52 & 0.54 \\
\hline 100 & FALSE & Outcome only & 0.10 & -0.48 & 0.78 & 0.84 & 0.79 & 0.97 \\
\hline 100 & FALSE & Single regression & -0.56 & -0.48 & 2.68 & 0.84 & 2.73 & 0.97 \\
\hline 400 & FALSE & DR g-estimator & 0.00 & -0.01 & 0.23 & 0.23 & 0.23 & 0.23 \\
\hline 400 & FALSE & Outcome only & 0.13 & -0.50 & 0.36 & 0.40 & 0.38 & 0.64 \\
\hline 400 & FALSE & Single regression & -0.62 & -0.50 & 1.23 & 0.40 & 1.37 & 0.64 \\
\hline 1600 & FALSE & DR g-estimator & 0.00 & -0.00 & 0.12 & 0.11 & 0.12 & 0.11 \\
\hline 1600 & FALSE & Outcome only & 0.12 & -0.51 & 0.19 & 0.20 & 0.22 & 0.55 \\
\hline 1600 & FALSE & Single regression & -0.60 & -0.51 & 0.65 & 0.20 & 0.88 & 0.55 \\
\hline 100 & TRUE & DR g-estimator & -0.02 & 0.01 & 0.69 & 0.52 & 0.69 & 0.52 \\
\hline 100 & TRUE & Outcome only & -0.29 & -0.53 & 1.35 & 0.58 & 1.38 & 0.79 \\
\hline 100 & TRUE & Single regression & -9.87 & -0.53 & 1.80 & 0.58 & 10.03 & 0.79 \\
\hline 400 & TRUE & DR g-estimator & -0.01 & -0.02 & 0.32 & 0.23 & 0.32 & 0.23 \\
\hline 400 & TRUE & Outcome only & -0.24 & -0.60 & 0.65 & 0.28 & 0.70 & 0.66 \\
\hline 400 & TRUE & Single regression & -9.89 & -0.60 & 0.96 & 0.28 & 9.94 & 0.66 \\
\hline 1600 & TRUE & DR g-estimator & -0.00 & -0.02 & 0.16 & 0.12 & 0.16 & 0.12 \\
\hline 1600 & TRUE & Outcome only & -0.25 & -0.61 & 0.33 & 0.15 & 0.42 & 0.63 \\
\hline 1600 & TRUE & Single regression & -9.92 & -0.61 & 0.49 & 0.15 & 9.94 & 0.63 \\
\hline
\end{tabular}

Note. The true values of $\psi_{0,0}$ and $\psi_{1,0}$ were 0.7 . All results were rounded to two decimal places. 


\section{Illustration}

In this section, we illustrate the introduced g-estimation procedure using a publicly available real-world dataset. Johannes et al. (2022) explored the effects of media usage on life satisfaction using data from a six-week longitudinal study (with weekly measurements) of a nationally representative cohort of people 16 years and older living in the United Kingdom. The researchers tested whether the use versus nonuse of specific media types in a particular week subsequently affected life satisfaction one week later. For illustration, we selected the use versus nonuse of books as a binary treatment of interest $(A)$. Life satisfaction was measured using two items "All things considered, how satisfied are you with your life as a whole nowadays?" and "To what extent do you feel that the things you do in your life are worthwhile?" Each item was rated on an eleven-point scale, ranging from 0 (not at all) to 10 (completely). Responses to both items were averaged to form a single indicator of life satisfaction (outcome $Y$ ). Further details of the study are provided in Johannes et al. (2022) 12

We used age, gender, time spent using each of the other media types (music, films, TV, games, magazines, and audiobooks), happiness, and anxiety as covariates (jointly denoted by $L$ ). The first two were time-invariant covariates measured only in the first wave, while the remaining were time-varying covariates measured at each wave. Happiness and anxiety were the responses to the items "How happy did you feel yesterday?" and "How anxious did you feel yesterday?" respectively; each was rated on an eleven-point scale, ranging from 0 (not at all) to 10 (completely). Because these covariates were the only plausible confounders recorded in the publicly available data set, we assumed for illustrative purposes that unconfoundedness was met given these covariates. Practical considerations on how researchers can improve the defensibility of the unconfoundedness

\footnotetext{
12 We utilized the processed dataset (Version 1 of the file processed_data.rds from https://osf.io/cv2u9/files/osfstorage/60747466f6585f051b61b85e) available as part of the project's Open Science Framework repository (https://osf.io/yn7sx/).
} 
assumption are described in the Discussion section.

We set the first wave as $t=0$ and started by considering the outcome at the final wave $T=5$ as the end-of-study outcome. For the purpose of illustrating the introduced g-estimation procedure, we focused on participants with complete data on the treatment, outcome, and covariates within each wave $(N=1974)$. Possible extensions to account for missing data are described in the Discussion section. The prevalence of book use (treatment) among these participants was $48 \%$ in the first wave $(t=0)$, then decreased gradually from $54 \%$ to $48 \%$ in the subsequent waves $(t=1,2,3,4)$. We posited the following linear SNMM to test possible effect modification at each time $t$ by Age and Anxiety:

$$
\begin{aligned}
& \mathrm{E}\left\{Y\left(\bar{a}_{t}, 0\right)-Y\left(\bar{a}_{t-1}, 0\right) \mid \bar{L}_{t}=\bar{l}_{t}, \bar{A}_{t-1}=\bar{a}_{t-1}\right\} \\
& =\left(\psi_{t, 0}+\psi_{t, 1} \text { Age }+\psi_{t, 2} \text { Anxiety }_{0}+\sum_{s=1}^{t} \psi_{t, s+2} \text { Anxiety }_{s}\right) a_{t} .
\end{aligned}
$$

The covariate Age was centered at its grand mean (across all individuals). To ease interpretation, we defined Anxiety as the individual difference from its baseline value (in the first recorded wave $t=0$ ) and included the baseline value ("Anxiety ${ }_{0}$ ") as a time-invariant covariate. Therefore, the causal parameter $\psi_{t, 2}$ encoded the effect of book use at time $t$ on the outcome (at time $T$ ) that depended on an individual's baseline anxiety level, whereas $\psi_{t, s+2}$ was the effect of book use at time $t$ due to a unit increase in an individual's anxiety level at time $s$ (where $1 \leq s \leq t$ ) from their baseline. We then carried out the introduced g-estimation procedure. For illustrative purposes, we fitted a propensity score model for the treatment at each time $t$ with main effects for the covariates' (and treatment's) histories. The outcome model included the main effects for the covariates' (and treatment's) histories, as well as the main effect of treatment and its interaction terms with the Age and Anxiety variables under the posited linear SNMM in (16). The nonparametric percentile bootstrap 95\% confidence intervals (CIs) were estimated using 10000 bootstrap samples with replacement. 
The g-estimators of the causal parameters encoding the effect of book use (treatment) at each time $t$ on life satisfaction (outcome) at time $T=5$ are shown in the rightmost columns of Table 2 (under the header " $T=5$ "). For example, the effect of book use at time $t=0$ on life satisfaction among individuals at the sample mean age with zero baseline anxiety levels $\left(\psi_{t=0,0}\right)$ was estimated to be $-0.39(95 \% \mathrm{CI}=(-0.78,-0.02))$. However, after adjusting for book use at time $t=0\left(A_{0}\right)$ as a baseline covariate, the effects of subsequent book use $(t>0)$ on life satisfaction were closer to zero or even positive; e.g., the corresponding effect of book use at $t=4$ on life satisfaction at $T=5\left(\psi_{t=4,0}\right)$ was estimated to be $0.15(-0.36,0.65)$.

We then carried out the g-estimation procedure focusing on the outcome at each wave $T=4,3,2,1$ in turn as the end-of-study outcome. These analyses were conducted using $N=1985,1996,2018$, and 2047 participants with complete data on the treatment, outcome, and covariates within each wave. We posited the same linear SNMM in (16) and carried out the g-estimation procedure as described above. The g-estimators of the causal effects on the life satisfaction outcome at each given time $T$ are similarly shown in Table 2 . We arrived at similar conclusions when focusing on the outcome at each wave $T=4,3,2,1$ in turn as those for the (final) outcome at time $T=5$. For example, after adjusting for book use at time $t=0\left(A_{0}\right)$ as a baseline covariate, the estimated effects of book use at a time $t>0$ on life satisfaction at a later time $T>t$ were negligible or weak at best.

In summary, in line with the conclusions of Johannes et al. (2022), which were drawn by assessing only a lag one (unconditional) association between book use and life satisfaction, there was negligible evidence that book use affected life satisfaction 13 Importantly, g-estimation of the causal parameters encoded in the posited linear SNMM in (16) provided much richer insights into the time-varying effects of book use, by estimating

\footnotetext{
13 In practice, researchers may consider using simulation-based power analyses at the study design stage to justify sufficient statistical power to detect (the presence of) specific effects of interest; see, e.g., Arnold et al. (2011) and Maxwell et al. (2007). We thank two anonymous reviewers for raising this point.
} 
different effects at each wave and investigating effect modification by time-invariant covariates (age and baseline anxiety) and a time-varying covariate (change in anxiety from baseline). 
Table 2

Estimates of the causal effects of book use at time $t$ on life satisfaction at time $T$ in the illustration. The point estimates

("Est."), nonparametric bootstrap standard errors ("SE"), and nonparametric percentile bootstrap 95\% CIs, are shown.

\begin{tabular}{|c|c|c|c|c|c|c|c|c|c|c|c|c|c|c|c|}
\hline \multirow[b]{2}{*}{ Effect } & \multicolumn{3}{|c|}{$T=1$} & \multicolumn{3}{|c|}{$T=2$} & \multicolumn{3}{|c|}{$T=3$} & \multicolumn{3}{|c|}{$T=4$} & \multicolumn{3}{|c|}{$T=5$} \\
\hline & Est. & $\mathrm{SE}$ & $95 \%$ CI & Est. & SE & $95 \%$ CI & Est. & $\mathrm{SE}$ & $95 \%$ CI & Est. & $\mathrm{SE}$ & $95 \%$ CI & Est. & $\mathrm{SE}$ & $95 \%$ CI \\
\hline$\psi_{0,0}$ & -29.8 & 9.8 & $(-49.0,-10.4)$ & -26.3 & 11.3 & $(-48.6,-3.8)$ & -42.6 & 12.8 & $(-67.3,-16.9)$ & -46.1 & 13.2 & $(-71.6,-19.9)$ & $\begin{array}{l}-39.1 \\
\end{array}$ & 19.3 & $(-77.7,-1.8)$ \\
\hline$\psi_{0,1}$ & 0.0 & 0.3 & $\left(\begin{array}{ll}-0.5, & 0.5)\end{array}\right.$ & 0.2 & 0.4 & $(-0.5,0.9)$ & -0.5 & 0.4 & $\left(\begin{array}{ll}-1.4, & 0.3)\end{array}\right.$ & 0.1 & 0.4 & $(-0.7, \quad 0.9)$ & -0.6 & 0.6 & $(-1.8,0.5)$ \\
\hline$\psi_{0,2}$ & 2.3 & 1.6 & $(-0.8, \quad 5.4)$ & 0.7 & 1.8 & $(-3.0,4.0)$ & 3.3 & 2.1 & $(-0.9, \quad 7.3)$ & 2.5 & 2.1 & $(-1.6, \quad 6.5)$ & 2.6 & 3.1 & $(-3.4,8.7)$ \\
\hline$\psi_{1,0}$ & & & & -0.5 & 11.6 & $(-23.6,22.4)$ & 1.2 & 17.1 & $(-30.9,35.7)$ & -39.0 & 19.2 & $(-74.8,-0.1)$ & -22.2 & 26.9 & $(-75.5,29.3)$ \\
\hline$\psi_{1,1}$ & & & & 0.1 & 0.3 & $(-0.5, \quad 0.8)$ & 0.6 & 0.5 & $(-0.4, \quad 1.6)$ & -0.6 & 0.6 & $(-1.7, \quad 0.6)$ & -0.3 & 0.8 & $(-2.0, \quad 1.2)$ \\
\hline$\psi_{1,2}$ & & & & 0.2 & 1.7 & $(-3.2,3.6)$ & -0.4 & 2.7 & $(-6.0, \quad 4.8)$ & 5.5 & 2.8 & $(-0.2,10.6)$ & 3.9 & 4.2 & $(-4.2,12.3)$ \\
\hline$\psi_{1,3}$ & & & & -0.4 & 2.6 & $(-5.5,4.8)$ & -4.8 & 3.2 & $(-11.1, \quad 1.1)$ & -3.1 & 4.1 & $(-10.9, \quad 5.0)$ & -7.8 & 5.6 & $(-18.4,3.3)$ \\
\hline$\psi_{2,0}$ & & & & & & & -9.9 & 16.0 & $(-43.1,19.7)$ & 40.8 & 19.3 & $(-1.4,74.9)$ & 3.6 & 27.3 & $(-54.5,53.5)$ \\
\hline$\psi_{2,1}$ & & & & & & & -0.3 & 0.5 & $\left(\begin{array}{ll}-1.2, & 0.7\end{array}\right)$ & 0.2 & 0.6 & $(-1.1, \quad 1.4)$ & -0.2 & 0.9 & $(-1.7,1.6)$ \\
\hline$\psi_{2,2}$ & & & & & & & 1.3 & 2.5 & $(-3.5, \quad 6.4)$ & -5.3 & 3.0 & $(-10.7, \quad 1.2)$ & -0.4 & 4.5 & $(-8.6,9.1)$ \\
\hline$\psi_{2,3}$ & & & & & & & -0.8 & 3.3 & $(-7.0, \quad 6.3)$ & 7.4 & 4.9 & $(-2.8,16.5)$ & -5.3 & 5.0 & $(-15.6,4.3)$ \\
\hline$\psi_{2,4}$ & & & & & & & 6.8 & 4.0 & $(-1.2,14.3)$ & -4.5 & 5.4 & $(-14.3, \quad 7.0)$ & 4.4 & 5.4 & $(-6.2,15.1)$ \\
\hline$\psi_{3,0}$ & & & & & & & & & & 8.6 & 15.9 & $(-22.4,39.7)$ & 2.1 & 25.4 & $(-45.9,53.8)$ \\
\hline$\psi_{3,1}$ & & & & & & & & & & 1.1 & 0.5 & $(0.0, \quad 1.9)$ & 1.4 & 0.6 & $(0.2, \quad 2.8)$ \\
\hline$\psi_{3,2}$ & & & & & & & & & & -2.1 & 2.4 & $(-6.8, \quad 2.6)$ & -2.1 & 3.8 & $(-9.8, \quad 5.2)$ \\
\hline$\psi_{3,3}$ & & & & & & & & & & -1.4 & 4.5 & $(-10.3, \quad 7.2)$ & 1.8 & 5.1 & $(-8.5,11.6)$ \\
\hline$\psi_{3,4}$ & & & & & & & & & & -1.5 & 5.3 & $(-11.6, \quad 9.3)$ & 4.7 & 5.2 & $(-5.9,14.7)$ \\
\hline$\psi_{3,5}$ & & & & & & & & & & 3.6 & 5.4 & $(-7.2,13.8)$ & -0.6 & 5.3 & $(-11.4,9.9)$ \\
\hline$\psi_{4,0}$ & & & & & & & & & & & & & 14.7 & 25.6 & $(-35.7,64.7)$ \\
\hline$\psi_{4,1}$ & & & & & & & & & & & & & -0.4 & 0.7 & $(-1.7,0.9)$ \\
\hline$\psi_{4,2}$ & & & & & & & & & & & & & -2.8 & 3.8 & $(-10.4,4.6)$ \\
\hline$\psi_{4,3}$ & & & & & & & & & & & & & 4.6 & 5.2 & $(-5.2,14.9)$ \\
\hline$\psi_{4,4}$ & & & & & & & & & & & & & -0.7 & 4.6 & $(-9.7,8.4)$ \\
\hline$\psi_{4,5}$ & & & & & & & & & & & & & -0.5 & 5.6 & $(-11.5,10.5)$ \\
\hline$\psi_{4,6}$ & & & & & & & & & & & & & 3.4 & 5.8 & $(-8.5,14.3)$ \\
\hline
\end{tabular}

Note. The causal parameters under the linear SNMM 16] encoding the effect of treatment at time $t$ on the outcome at time $T$ are indexed by $\psi_{t, s}, s=0, \ldots, t+2$.

Explanations of the causal parameters are provided in the main text following [16]. Blank spaces correspond to ill-defined effects where $t \geq T$. All results were multiplied by 100 and then rounded to one decimal place. 


\section{Discussion}

In this article, we introduced a g-estimation strategy for time-varying treatments in the presence of time-varying confounding (Vansteelandt \& Sjölander, 2016). We believe g-estimation has immense potential for improving causal inferences of longitudinal data in psychological research because of its various appealing features. Effect modification by time-invariant and time-varying covariates can be readily investigated using covariate-treatment interactions under the specified linear SNMM. Covariates can be either continuous or noncontinuous and be either time-invariant or time-varying - and thus possibly affected by treatment - with no models for the (distributions of the) covariates required for estimation. Continuous and noncontinuous treatments can be accommodated using any propensity score model for the treatment. Under a correctly-specified linear SNMM describing the treatment effect on the (potential) outcomes, and assuming that unconfoundedness holds, the g-estimator is consistent for the treatment effects when the propensity score model or outcome model is correctly specified, but not necessarily both.

\section{Practical considerations for implementing g-estimation}

Researchers should consider a few practical issues before implementing the introduced g-estimation strategy. First, a central challenge of drawing causal inferences for non-randomized treatments is selecting covariates that can satisfy unconfounded assumptions (Steiner et al., 2010). Ideally, during the study design phase, researchers should compile a rich and comprehensive selection of relevant treatment-outcome confounders based on established subject matter theory or extant empirical evidence. The confounders should then be recorded at baseline, and if they are time-varying and possibly affected by treatment, recorded at intermediate waves as well. These confounders $L$ may

share unmeasured common causes with the outcome(s), such as $U$ in Figure 1. We reiterate that no models for the time-varying confounders, e.g., given their causally and temporally antecedent predictors (such as earlier instances of treatment or outcome, or both), are required for constructing g-estimators of the time-varying effects. 
Second, researchers must deliberately determine the most appropriate SNMM that describes the time-varying treatment effects of substantive interest. For example, would an SNMM with a single causal effect at each time $t$, such as (6), suffice? Or are the researchers interested in investigating effect modification by either time-invariant or time-varying covariates, such as $(16)$ in the applied example?

Third, researchers must decide on the propensity score and outcome models used to carry out the g-estimation procedure. While the introduced g-estimator is consistent when either the propensity score or the outcome model is correctly specified (and unbiased when both are correctly specified), it is still susceptible to biases - as are all methods for estimating causal effects - when both models are incorrectly specified. For example, researchers should carefully consider the number of prior time lags for the predictors in each estimation model. Following Cole and Maxwell (2003), we advise including both lag one and lag two effects, and possibly longer lags if there is sufficient complete data across consecutive waves, to permit the possibility of more complex relations among the predictors. A related consideration is deciding the functional form of the predictors. For example, the possibility of including more complex higher-order or interaction terms among the predictors, such as treatment-confounder or confounder-confounder interactions, in the estimation models should be examined. Thoughtful considerations of these questions before carrying out the g-estimation procedure strengthen the validity of causal inferences drawn using longitudinal data.

\section{Limitations and future work}

In practice, participants in longitudinal studies frequently complete some questions but not others, even within the same wave. For illustrative purposes, we made the simplification of excluding all observations with partially recorded data from the analysis. A direction for future work is to leverage all available information in an incomplete data set by incorporating missing data methods, such as multiple imputations by chained equations (Azur et al., 2011), iterative robust model-based imputation (Templ et al., 2011), 
or substantive model compatible fully conditional specifications (Bartlett et al., 2015).

In this paper, we used standard maximum likelihood estimation to fit the propensity score models and to calculate the predicted propensity scores entered into the outcome model. We encourage future work to explore alternative approaches to predict the propensity scores, such as methods leveraging data adaptive or machine learning algorithms to reduce the reliance on parametric models (Lee et al., 2010). For example, covariate balancing propensity scores (Imai \& Ratkovic, 2014) utilize a parametric functional form of a logistic regression model, but directly maximize the balance of the covariate distributions across different treatment groups when determining the optimal (propensity score) model parameter values. Another example is generalized boosted models (McCaffrey et al., 2004, Setodji et al., 2017) that do not require assuming a parametric (propensity score) model and utilize novel data-adaptive algorithms designed to detect non-linear relationships to achieve covariate balance. The simulation-based performances of these methods could be compared under more extensive and complex data-generating scenarios (see, e.g., Setodji et al., 2017) in future neutral comparison studies (Boulesteix et al., 2018).

The validity of inferences about the causal effects is predicated on assuming that the unconfoundedness of the treatment-outcome association at a particular time point can be reasonably met. Therefore, it is judicious to conduct a sensitivity analysis addressing unmeasured confounding by judging the (minimum) extent to which substantive conclusions would differ after adjusting for an additional hypothetical unmeasured confounder; see Liu et al. (2013) for an overview of such analyses. In principle, one can implement existing methods for treatments (and outcomes) at a single time point, such as Carnegie et al. (2016) and VanderWeele and Ding (2017), to the treatment-outcome association at each time point in turn to assess the impact of unmeasured confounding.

\section{Conclusion}

In observational studies with non-randomized treatments, valid statistical inferences of causal effects are contingent on adjusting for baseline or pre-treatment confounders of 
treatment and outcome. But when longitudinal study designs are utilized - as are often in psychological research - repeated measurements of the covariates, treatment, or outcome at different time points or waves inevitably induce time-varying confounding of the causal effects. Adjusting for post-treatment or time-varying confounding is difficult because merely including or omitting such confounders can, either way, lead to incorrect inferences. We have introduced a g-estimation strategy under linear SNMMs previously developed in the causal inference literature to avoid this situation (Hernán \& Robins, 2020). Implementation is straightforward using standard regression functions widely utilized by applied researchers. The resulting g-estimators have a double robustness property that ensures their unbiasedness even under certain model misspecifications. In summary, g-estimation is flexible, effective, robust, and relatively straightforward to implement. We encourage psychology researchers to employ the g-estimation strategy when testing the causal effects of a time-varying treatment in longitudinal studies. 


\section{References}

Arnold, B. F., Hogan, D. R., Colford, J. M., \& Hubbard, A. E. (2011). Simulation methods to estimate design power: An overview for applied research. BMC Medical Research Methodology, 11(1), 94. https://doi.org/10.1186/1471-2288-11-94

Azur, M. J., Stuart, E. A., Frangakis, C., \& Leaf, P. J. (2011). Multiple imputation by chained equations: What is it and how does it work? International Journal of Methods in Psychiatric Research, 20(1), 40-49.

https://doi.org/https://doi.org/10.1002/mpr.329

Bang, H., \& Robins, J. M. (2005). Doubly robust estimation in missing data and causal inference models. Biometrics, 61(4), 962-973.

Baron, R. M., \& Kenny, D. A. (1986). The moderator-mediator variable distinction in social psychological research: Conceptual, strategic, and statistical considerations. Journal of Personality and Social Psychology, 51(6), 1173. https://doi.org/10.1037/0022-3514.51.6.1173

Bartlett, J. W., Seaman, S. R., White, I. R., \& Carpenter, J. R. (2015). Multiple imputation of covariates by fully conditional specification: Accommodating the substantive model [PMID: 24525487]. Statistical Methods in Medical Research, 24 (4), 462-487. https://doi.org/10.1177/0962280214521348

Boulesteix, A.-L., Binder, H., Abrahamowicz, M., Sauerbrei, W., \&

for the Simulation Panel of the STRATOS Initiative. (2018). On the necessity and design of studies comparing statistical methods. Biometrical Journal, 60(1), 216-218. https://doi.org/10.1002/bimj.201700129

Carnegie, N. B., Harada, M., \& Hill, J. L. (2016). Assessing sensitivity to unmeasured confounding using a simulated potential confounder. Journal of Research on Educational Effectiveness, 9(3), 395-420.

https://doi.org/10.1080/19345747.2015.1078862 
Cole, D. A., \& Maxwell, S. E. (2003). Testing mediational models with longitudinal data: Questions and tips in the use of structural equation modeling. Journal of Abnormal Psychology, 112(4), 558-577. https://doi.org/10.1037/0021-843X.112.4.558

D’Agostino Jr, R. B. (1998). Propensity score methods for bias reduction in the comparison of a treatment to a non-randomized control group. Statistics in medicine, 17(19), 2265-2281.

Daniel, R. M., Cousens, S., De Stavola, B., Kenward, M. G., \& Sterne, J. A. C. (2013). Methods for dealing with time-dependent confounding. Statistics in Medicine, 32(9), 1584-1618. https://doi.org/https://doi.org/10.1002/sim.5686

Ding, P., \& Miratrix, L. W. (2015). To adjust or not to adjust? sensitivity analysis of m-bias and butterfly-bias. Journal of Causal Inference, 3(1), 41-57. https://doi.org/doi:10.1515/jci-2013-0021

Duncan, O. D. (1966). Path analysis: Sociological examples. American Journal of Sociology, 72(1), 1-16. https://doi.org/10.1086/224256

Efron, B., \& Tibshirani, R. J. (1994). An introduction to the bootstrap. Chapman; Hall/CRC. https://doi.org/10.1007/978-1-4899-4541-9

Elwert, F. (2013). Graphical causal models. In S. L. Morgan (Ed.), Handbook of causal analysis for social research (pp. 245-273). Springer Netherlands. https://doi.org/10.1007/978-94-007-6094-3_13

Elwert, F., \& Winship, C. (2014). Endogenous selection bias: The problem of conditioning on a collider variable [PMID: 30111904]. Annual Review of Sociology, 40(1), 31-53. https://doi.org/10.1146/annurev-soc-071913-043455

Ghosh, S., \& Tan, Z. (2022). Doubly robust semiparametric inference using regularized calibrated estimation with high-dimensional data. Bernoulli, 28(3), 1675-1703. https://doi.org/10.3150/21-BEJ1378

Glymour, M. M., Jewell, N. P., \& Pearl, J. (2016). Causal inference in statistics: A primer. John Wiley \& Sons Ltd. 
Greenland, S. (2003). Quantifying biases in causal models: Classical confounding vs collider-stratification bias. Epidemiology, 14(3), 300-306. http://www.jstor.org/stable/3703850

Grosz, M. P., Rohrer, J. M., \& Thoemmes, F. (2020). The taboo against explicit causal inference in nonexperimental psychology [PMID: 32727292]. Perspectives on Psychological Science, 15(5), 1243-1255. https://doi.org/10.1177/1745691620921521

Hernán, M. A., \& Robins, J. M. (2020). Causal inference: What if. Chapman \& Hall \CRC.

Hirano, K., \& Imbens, G. W. (2004). The propensity score with continuous treatments. Applied Bayesian modeling and causal inference from incomplete-data perspectives, 226164, 73-84.

Hittner, E. F., Stephens, J. E., Turiano, N. A., Gerstorf, D., Lachman, M. E., \& Haase, C. M. (2020). Positive affect is associated with less memory decline: Evidence from a 9-year longitudinal study [PMID: 33090935]. Psychological Science, 31 (11), 1386-1395. https://doi.org/10.1177/0956797620953883

Imai, K., \& Ratkovic, M. (2014). Covariate balancing propensity score. Journal of the Royal Statistical Society: Series B (Statistical Methodology), 76(1), 243-263. https://doi.org/https://doi.org/10.1111/rssb.12027

Imbens, G. W., \& Rubin, D. B. (2015). Causal inference in statistics, social, and biomedical sciences. Cambridge University Press.

Johannes, N., Dienlin, T., Bakhshi, H., \& Przybylski, A. K. (2022). No effect of different types of media on well-being. Scientific Reports, 12(1), 61. https://doi.org/10.1038/s41598-021-03218-7

Kang, J. D., \& Schafer, J. L. (2007). Demystifying double robustness: A comparison of alternative strategies for estimating a population mean from incomplete data. Statistical science, 22(4), 523-539.

Keogh, R. H., Daniel, R. M., VanderWeele, T. J., \& Vansteelandt, S. (2017). Analysis of Longitudinal Studies With Repeated Outcome Measures: Adjusting for 
Time-Dependent Confounding Using Conventional Methods. American Journal of Epidemiology, 187(5), 1085-1092. https://doi.org/10.1093/aje/kwx311

Lee, B. K., Lessler, J., \& Stuart, E. A. (2010). Improving propensity score weighting using machine learning. Statistics in Medicine, 29(3), 337-346. https://doi.org/https://doi.org/10.1002/sim.3782

Liu, W., Kuramoto, S. J., \& Stuart, E. A. (2013). An introduction to sensitivity analysis for unobserved confounding in nonexperimental prevention research. Prevention science, $14(6), 570-580$.

Maxwell, S. E., Kelley, K., \& Rausch, J. R. (2007). Sample size planning for statistical power and accuracy in parameter estimation. Annual Review of Psychology, 59(1), 537-563. https://doi.org/10.1146/annurev.psych.59.103006.093735

doi: 10.1146/annurev.psych.59.103006.093735

McCaffrey, D. F., Ridgeway, G., \& Morral, A. R. (2004). Propensity score estimation with boosted regression for evaluating causal effects in observational studies. Psychological methods, 9(4), 403.

Naimi, A. I., Cole, S. R., \& Kennedy, E. H. (2016). An introduction to g methods. International Journal of Epidemiology, 46(2), 756-762. https://doi.org/10.1093/ije/dyw323

Newsome, S. J., Keogh, R. H., \& Daniel, R. M. (2018). Estimating long-term treatment effects in observational data: A comparison of the performance of different methods under real-world uncertainty. Statistics in Medicine, 37(15), 2367-2390. https://doi.org/https://doi.org/10.1002/sim.7664

Nye, C. D., Wille, B., Amory, J., \& De Fruyt, F. (2021). Are work activities related to interest change over time? A 22-year longitudinal study. Journal of Personality and Social Psychology, 121(4), 865-893. https://doi.org/10.1037/pspp0000360 
Pearl, J. (2010). On the consistency rule in causal inference: Axiom, definition, assumption, or theorem? Epidemiology, 21(6), 872-875. https://doi.org/10.1097/EDE.0b013e3181f5d3fd

R Core Team. (2021). R: A language and environment for statistical computing. $\mathrm{R}$ Foundation for Statistical Computing. Vienna, Austria. https://www.R-project.org/

Ren, D., Stavrova, O., \& Loh, W. W. (2022). Nonlinear effect of social interaction quantity on psychological well-being: Diminishing returns or inverted u? Journal of Personality and Social Psychology, 122(6), 1056-1074. https://doi.org/10.1037/pspi0000373

Robins, J. M. (1994). Correcting for non-compliance in randomized trials using structural nested mean models. Communications in Statistics-Theory and methods, 23(8), 2379-2412. https://doi.org/10.1080/03610929408831393

Robins, J. M. (2000a). Marginal structural models versus structural nested models as tools for causal inference. In M. E. Halloran \& D. Berry (Eds.), Statistical models in epidemiology, the environment, and clinical trials (pp. 95-133). Springer-Verlag. https://doi.org/10.1007/978-1-4612-1284-3_2

Robins, J. M. (2000b). Robust estimation in sequentially ignorable missing data and causal inference models. Proceedings of the American Statistical Association, 1999, 6-10.

Rosenbaum, P. R. (1984). The consquences of adjustment for a concomitant variable that has been affected by the treatment. Journal of the Royal Statistical Society. Series A (General), 147(5), 656-666. https://doi.org/10.2307/2981697

Rosenbaum, P. R., \& Rubin, D. B. (1983). The central role of the propensity score in observational studies for causal effects. Biometrika, $70(1), 41-55$.

Rubin, D. B. (1986). Comment: Which ifs have causal answers. Journal of the American statistical association, 81(396), 961-962.

Setodji, C. M., McCaffrey, D. F., Burgette, L. F., Almirall, D., \& Griffin, B. A. (2017). The right tool for the job: Choosing between covariate-balancing and generalized 
boosted model propensity scores. Epidemiology, 28(6), 802-811.

https://doi.org/10.1097/EDE.0000000000000734

Steiner, P. M., Cook, T. D., Shadish, W. R., \& Clark, M. H. (2010). The importance of covariate selection in controlling for selection bias in observational studies. Psychological Methods, 15(3), 250-267. https://doi.org/10.1037/a0018719

Templ, M., Kowarik, A., \& Filzmoser, P. (2011). Iterative stepwise regression imputation using standard and robust methods. Comput. Stat. Data Anal., 55(10), 2793-2806. https://doi.org/10.1016/j.csda.2011.04.012

Thoemmes, F. (2015). M-bias, butterfly bias, and butterfly bias with correlated causes - a comment on Ding and Miratrix (2015). Journal of Causal Inference, 3(2), 253-258. https://doi.org/doi:10.1515/jci-2015-0012

VanderWeele, T. J., \& Ding, P. (2017). Sensitivity analysis in observational research: Introducing the E-value. Annals of internal medicine.

Vansteelandt, S., \& Daniel, R. M. (2014). On regression adjustment for the propensity score. Statistics in Medicine, 33(23), 4053-4072. https://doi.org/10.1002/sim.6207

Vansteelandt, S., \& Joffe, M. (2014). Structural nested models and G-estimation: The partially realized promise. Statistical Science, 29(4), 707-731. https://doi.org/10.1214/14-STS493

Vansteelandt, S., \& Sjölander, A. (2016). Revisiting G-estimation of the effect of a time-varying exposure subject to time-varying confounding. Epidemiologic Methods, 5(1), 37-56. https://doi.org/10.1515/em-2015-0005

Wright, S. (1934). The method of path coefficients. The Annals of Mathematical Statistics, 5(3), 161-215. https://doi.org/10.1214/aoms/1177732676 